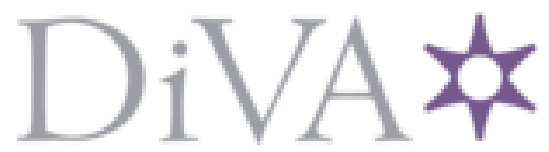

http://www.diva-portal.org

\title{
Postprint
}

This is the accepted version of a paper published in Physics Teacher. This paper has been peerreviewed but does not include the final publisher proof-corrections or journal pagination.

Citation for the original published paper (version of record):

Melander, E., Haglund, J., Weiszflog, M., Andersson, S. (2016)

More than meets the eye: Infrared cameras in open-ended university thermodynamics labs.

Physics Teacher, 54(9): 528-531

Access to the published version may require subscription.

N.B. When citing this work, cite the original published paper.

Permanent link to this version:

http://urn.kb.se/resolve?urn=urn:nbn:se:uu:diva-306683 


\section{More than meets the eye - infrared cameras in open-ended university thermodynamics labs}

Emil Melander, Jesper Haglund, Matthias Weiszflog, Staffan Andersson

Educational research has found that students have challenges understanding thermal science. ${ }^{1}$ Undergraduate physics students have difficulties differentiating basic thermal concepts, such as heat, temperature, and internal energy. ${ }^{2}$ Engineering students have been found to have difficulties grasping surface emissivity as a thermal material property. ${ }^{3}$ One potential source of students' challenges with thermal science is the lack of opportunity to visualize energy transfer in intuitive ways with traditional measurement equipment. Thermodynamics laboratories have typically depended on point measures of temperature by use of thermometers (detecting heat conduction) or pyrometers (detecting heat radiation). In contrast, thermal imaging by means of an infrared (IR) camera provides a real-time, holistic image. Here we provide some background on IR cameras and their uses in education, and summarize five qualitative investigations that we have used with our students.

Watching surfaces of for example metal, glass, or a whiteboard with an IR camera gives unprecedented opportunities to get an intuitive idea of their properties in relation to electromagnetic radiation, including emission, absorption, reflection and transparency at different wavelengths. As another application, students can quickly get an overview of complex thermodynamics laboratory apparatus, such as a heat pump, and identify its main components, i.e. condenser, evaporator and compressor (Figure 1 ). Then again, IR cameras will not address all issues in thermal teaching. For instance, even though students can see temperature changes involved in heat pumps, they are not helped in understanding the centrality of the phenomenon of phase changes inside the apparatus, which has been found to be another challenge on the topic. ${ }^{4}$
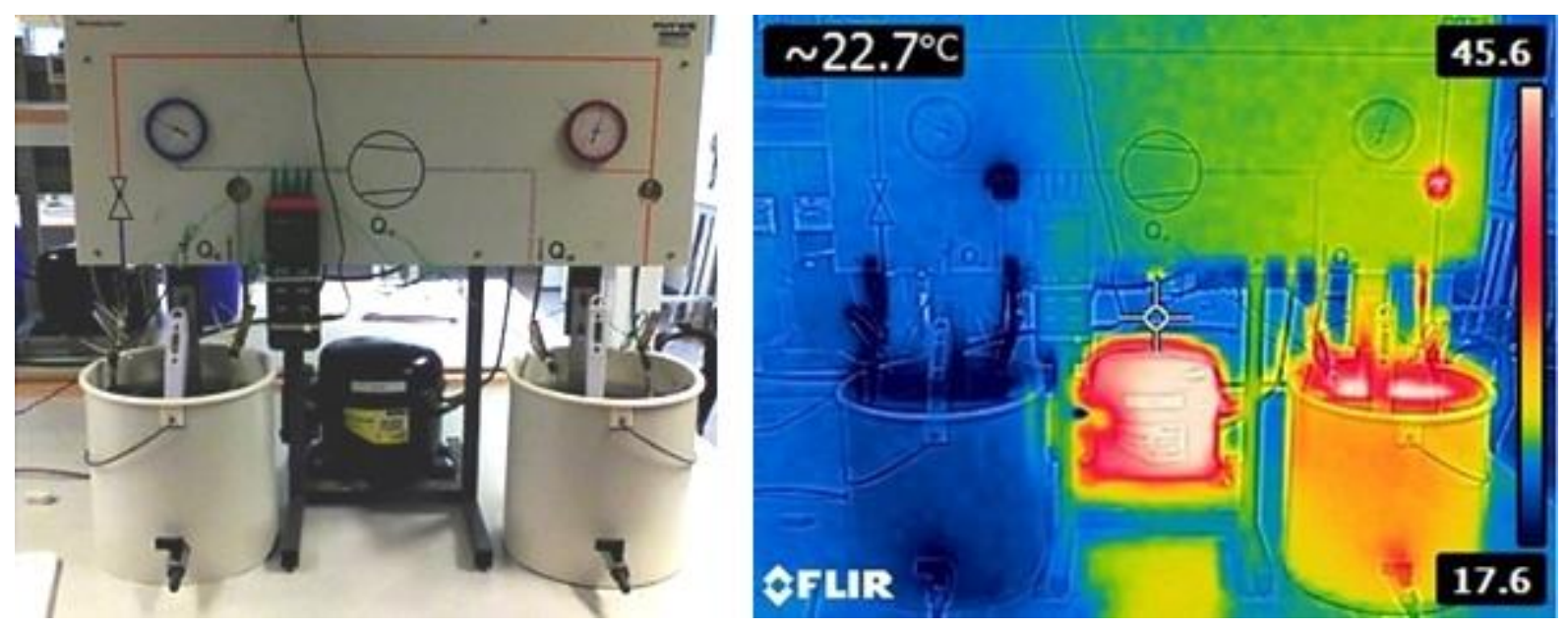

Fig. 1. A photo and a corresponding IR image of a heat pump with a compressor at work in the center with a heat source at low temperature (evaporator) to the left and a heat sink (condenser) at high temperature to the right. In the IR image, temperatures are rendered as different colors. Since the IR cameras can be set to take two pictures simultaneously, a thermal as well as a normal photograph, comparisons can be made 
between them. The temperature of the surface towards which the crosshair is directed is displayed as a number at top left of the screen.

Laboratory exercises are recognised as central in science teaching, but are often introduced in courses without much consideration about the purpose, and with meagre learning outcomes. ${ }^{5,6}$ In traditional expository labs, students follow a given procedure in order to reach a predetermined outcome, by deductive application of a general principle, in a cookbook fashion. ${ }^{7}$ The expository lab is suitable in order to attain certain learning goals, such as coming to master advanced laboratory equipment and procedures. However, if the goal is for students to develop conceptual understanding or problem-solving skills, or put their creativity to test, other more open-ended approaches might be worth considering. These include inquiry approaches, guided by students' own questions, or discovery and problem-based approaches, where students are given more autonomy in how to investigate predetermined phenomena. ${ }^{7}$ Here, the challenge is to provide sufficient guidance in written instructions and in student-teacher dialogue, without stifling students' ownership of their learning.

Vollmer and colleagues have proposed a wide range of phenomena that can be studied by means of IR cameras. ${ }^{8,9}$ This includes, but is not limited to thermal science, mechanics (e.g. inelastic collisions and friction), electric circuits, and electronics manufacturing. Xie and colleagues see the IR camera as a versatile thermal looking-glass that is particularly well suited for inquiry-based approaches to physics teaching. By using an IR camera, students will attend to and discover thermal phenomena that otherwise would have gone unnoticed. ${ }^{10,11}$ Haglund and colleagues have conducted a series of studies on grade 4-12 students' use of IR cameras in physics teaching. ${ }^{12-14}$ In practical predict-observe-explain exercises, ${ }^{15}$ students have focused on heat conduction, and the conundrum that metals feel cool in room temperature. Apart from providing the opportunity to seeing taught thermal phenomena, students have been found to engage in 'instant inquiry' with IR cameras, in which they ask 'what-ifs' and get immediate response in real time.

\section{Basic facts of IR cameras}

Thermal imaging relies on the phenomenon that matter emits electromagnetic radiation. The spectrum of the radiation depends on the temperature according to Planck's law. The sun, with a temperature of about $6000 \mathrm{~K}$ emits radiation mainly in the visible range $(400-750 \mathrm{~nm})$, which is detectible with a normal camera. For most everyday objects, in contrast, the radiation is primarily in the IR range. IR cameras detect IR radiation (typically in the range 3.0 $-14 \mu \mathrm{m}$ ), and renders the surface temperature, given assumptions of the emissivity $\varepsilon$. In the current study, we used handheld IR cameras (FLIR C2, E4 and E6) in the price range of $\$ 500-\$ 1500$, with $\varepsilon=$ 0.95 . Recently, IR camera functionality has been made available as mobile phone addons at lower prices, or integrated in the mobile phones themselves. The price depends strongly on the image resolution requirements.

We have designed a set of open laboratory assignments where IR cameras give added value in second year university thermodynamics courses for 
physics and engineering students, as a complement to predominately algebraic problem solving and lectures. The purpose of the assignments is to strengthen students' qualitative understanding of thermal concepts. In this regard, students are explicitly instructed not to make quantitative measurements, but to focus on providing underlying explanations of the phenomena. The assignments typically cover two hours each and are followed by class seminars with presentations for assessment. During the students' interaction with the assignments a lot of traditional thermodynamics lab equipment is available but there is only limited information for the students on how to use it. Students choose one of eight central concepts within the course and they are free to explore them as they like in order to present them on the seminar with an audience of their peers. This article highlights five of these concepts, where IR cameras have been found particularly helpful in open-ended inquiry-based laboratory assignments. We also describe briefly how students typically engage with the assignments, and reflect on limitations with the technology from an educational point of view.

\section{Five IR-camera-enhanced assignments}

Below, we introduce five examples of laboratory assignments where IR cameras have been utilized in our courses. The first three assignments focus on the functionality of experimental apparatus, and the last two involve open-ended investigation of thermal radiation as a phenomenon.

1. The components of heat pumps

This assignment involves a heat pump with two buckets of water that correspond to the heat reservoir and heat sink. The thermodynamic cycle involved in a heat pump allows heat transfer from a system of lower temperature to a system of higher temperature - against its spontaneous direction - by performing work. With thermal cameras one immediately gets a good overview of the components and their function (see Figure 1). The heat pump is often introduced theoretically in physics courses, so that students rarely get a chance to get acquainted with its technology and centrality of phase changes of the refrigerant. ${ }^{4}$ The process of heat transfer from a colder object to a warmer object is readily recognized by students as the functionality of a fridge.

2. Entropy changes in rubber bands

Entropy is a physical quantity that relates to the arrow of time since a spontaneous process always involves an entropy increase. Entropy is yet another thermodynamics concept that is challenging for students to grasp. ${ }^{16}$ In a typical metal, an increase in temperature results in an expanding material, due to the energy added to the crystalline bonds. In contrast, by controlling the temperature of an elastic rubber band an increased temperature yields a counterintuitive decrease in volume since the cross-linked polymer molecules behave as entropic springs with a spring constant that increases with temperature. Correspondingly expansion of said rubber band causes a temperature increase. In 
thermodynamics labs, with access to thermometers only, students have traditionally been encouraged to perceive the temperature changes of the rubber qualitatively by touching the band with their upper lips. ${ }^{17}$ In contrast, IR cameras are well suited for measurement of such rapid changes of surface temperatures. In our experience, stretching balloons gives clear temperature increases (See Figure 2).
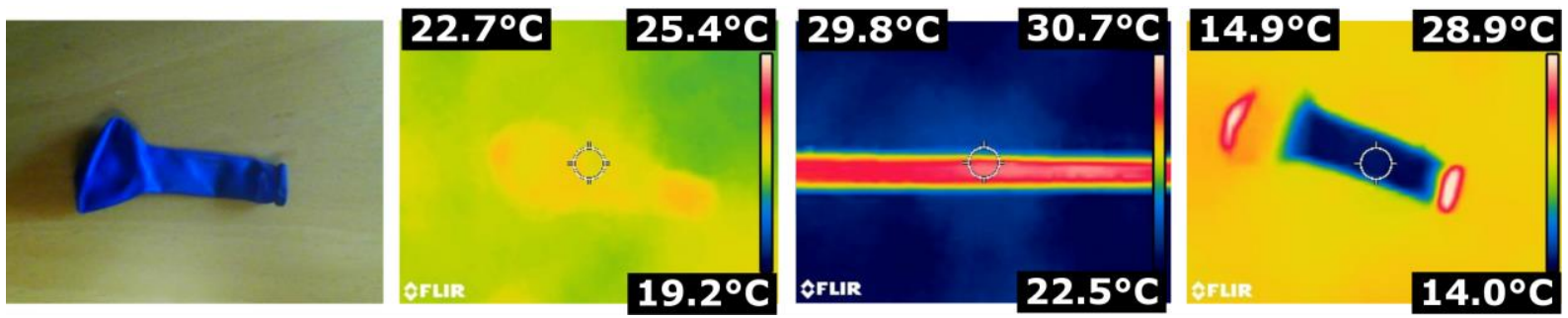

Fig. 2. A time series of the stretching of a balloon. The two images to the left show the balloon at room temperature. In the next image, the temperature increases as the balloon is stretched. The stretched balloon is allowed to cool to room temperature. The final image shows that the temperature of the balloon decreases below room temperature, as the tension is released. The two white areas by the ends indicate heat conduction from our hands.

\section{Cooling through Joule-Thomson throttling}

Joule-Thomson cooling (commonly known as throttling) is a process that occurs when a gas is forced through a porous plug or valve, and allowed to expand adiabatically with no heat exchange to its surroundings. This can be tested through checking the temperatures of the surroundings and the container of a $\mathrm{CO}_{2}$ fire extinguisher, which will be significantly cooler upon the release of the gas (see Figure 3 ). The temperature decrease is caused primarily by the phase change from liquid to gas state in the container as gas is released, during which energy is required to break bonds in the liquid $\mathrm{CO}_{2}$. In our experience, however, in line with Georgiou et al. ${ }^{18}$ students often fail to recognise the centrality of the phase change in the process. It should be noted here that the types of handheld IR cameras we used detect radiation primarily from solid and liquid surfaces, but not from gases, due to their low density. The low temperature readings depend on aerosol formation and cooling of the floor.
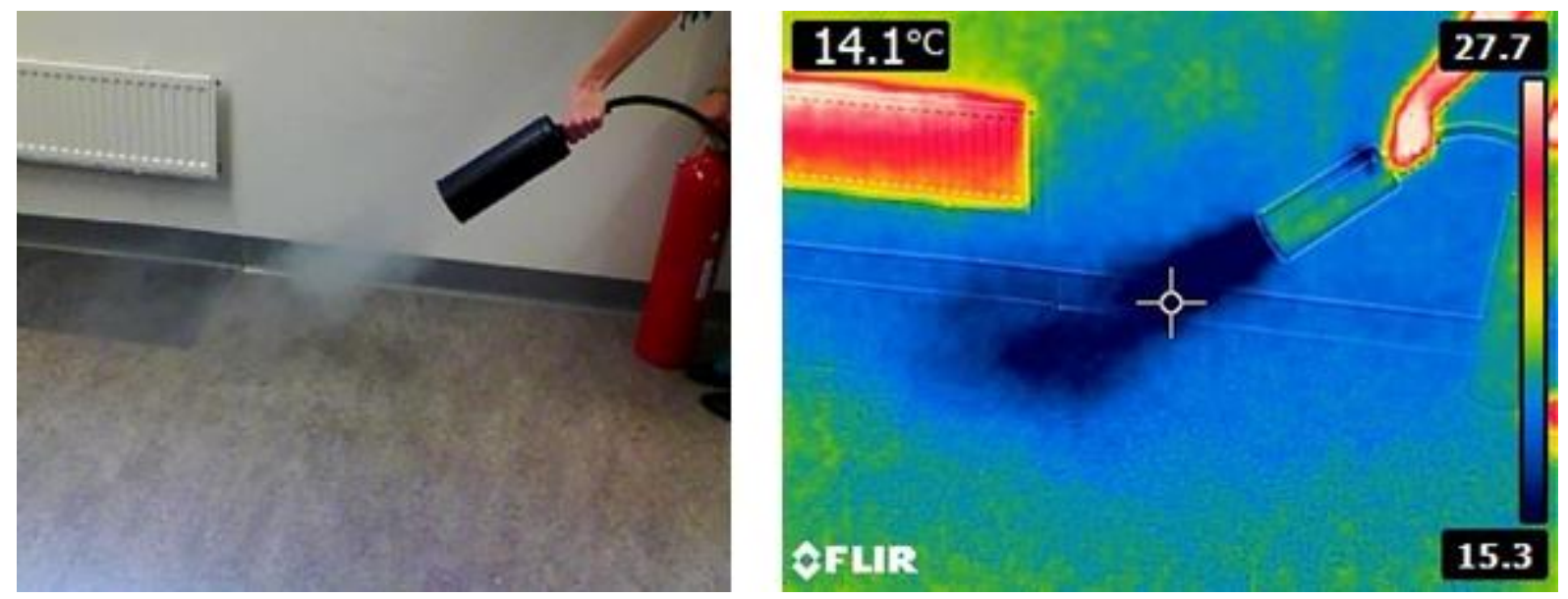

Fig. 3. A fire extinguisher where one can study the temperature decrease associated with releasing $\mathrm{CO}_{2}$ from the container. 


\section{Thermal radiation}

One of the assignments is for students to learn more about thermal radiation as a phenomenon. Students are generally referred to start by having a look at a Leslie's cube ${ }^{19}$ which is a metal cube with uniform temperature sustained by an internal heat source (typically a lamp or boiled water), whose four different surfaces have different material properties. ${ }^{9,20}$ In our case, there is a white- and a black-painted side, and two metal sides that are oxidized and polished, respectively, for student comparison of emissivity differences. As seen in Figure 4, the polished aluminum surface to the right has lower emissivity than the black-painted surface to the left, as revealed by the lower temperature readings. As opposed to pyrometers, IR cameras allow measurement of an entire surface at once, so that also reflections of thermal radiation from the surroundings will be clearly visible. Students are often intrigued to find that the emissivity of the white surface is very high and roughly equal to that of the black surface, both therefore being close to ideal black bodies in the IR range. ${ }^{21}$

Apart from contributing to students' qualitative understanding, IR cameras can also be used for quantitative measurement and modeling of emission, absorption and reflection of thermal radiation in interaction with a Leslie's cube. For instance, students can make use of Stefan-Boltzmann's law, or Kirschhoff's radiation law, i.e. that the absorptivity and emissivity of a surface are equal at a given wavelength.
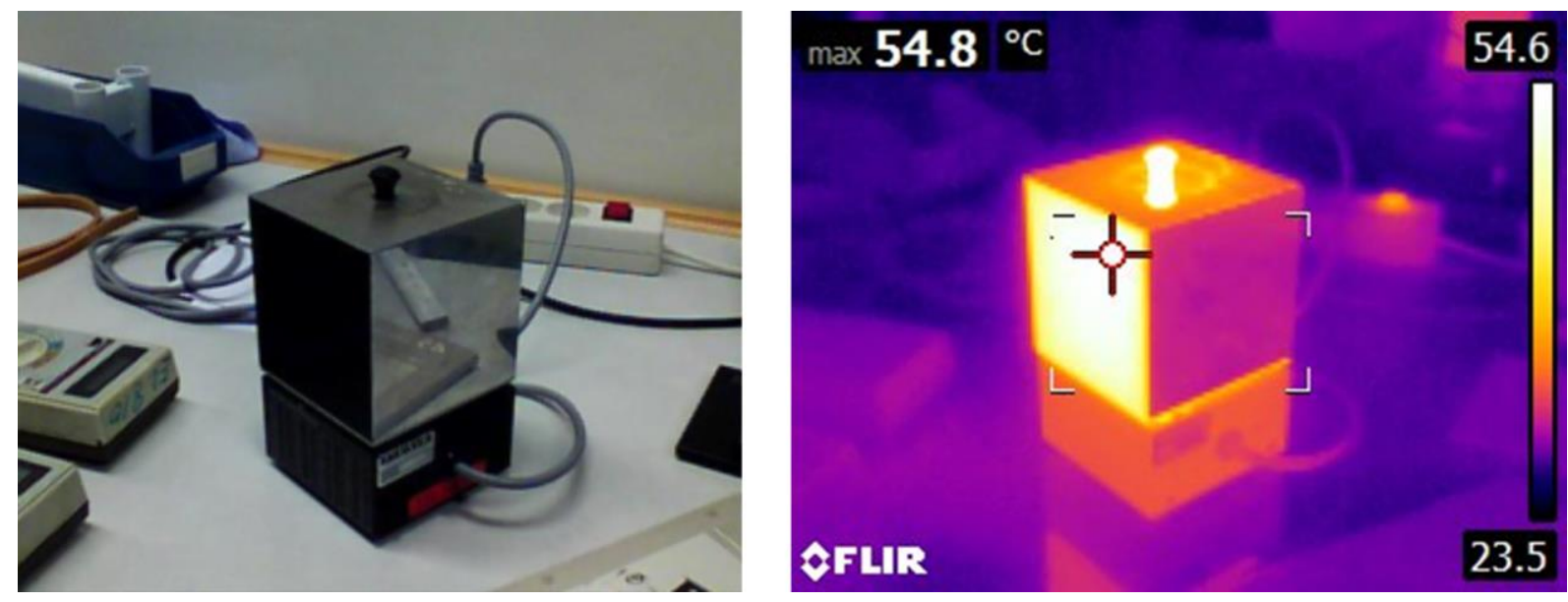

Fig. 4. A Leslie's cube can be used to study electromagnetic emissions and reflections from different surfaces.

5. Thermal imaging

Our most open-ended assignment involves investigation of the IR camera technology itself. What can be seen with thermal cameras and what cannot be seen, and why is there a difference in comparison with an ordinary camera detecting visible light? By allowing students to explore the lab and the university premises, they attend to relevant thermal phenomena, such as temperature decreases due to evaporation and 
temperatures of electronic equipment being higher than the surroundings. Students tend to be intrigued by seeing that whiteboards and windows reflect radiation in the IR range, but that a significant proportion of IR radiation penetrates a balloon, in contrast to the behavior of visual light (see Figure 5).
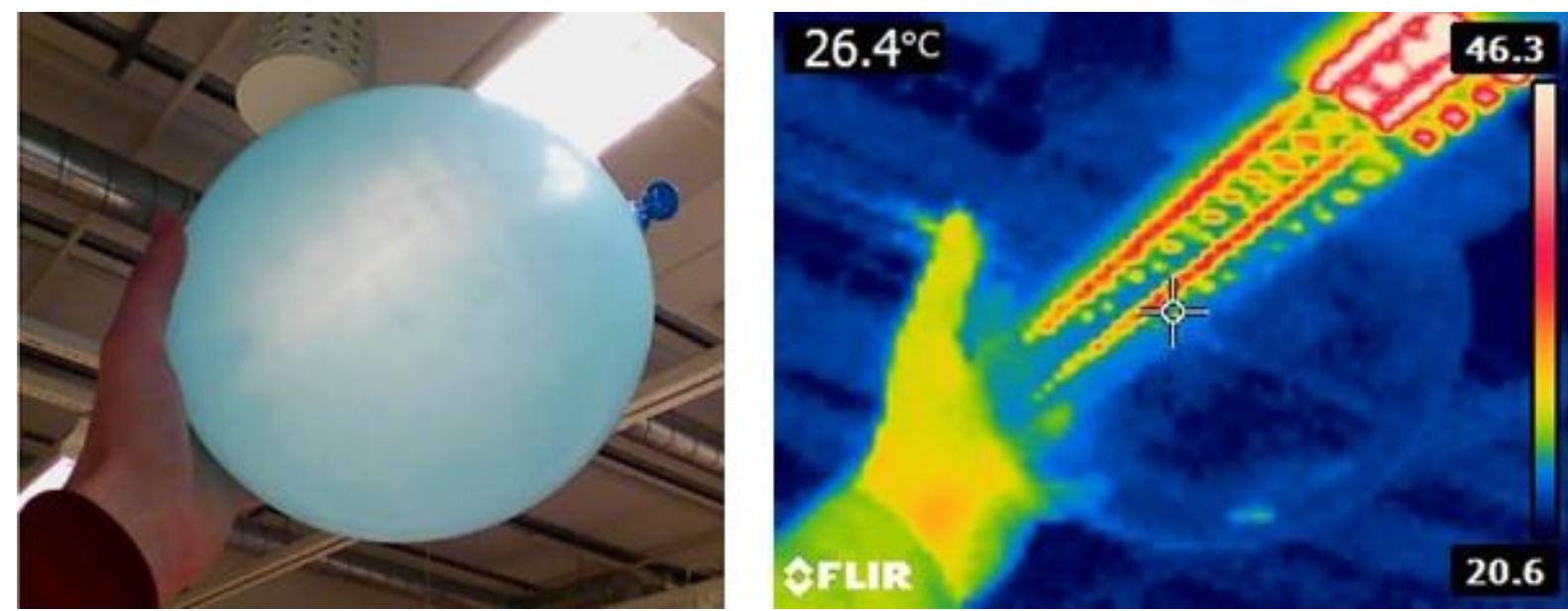

Fig. 5. IR radiation can be seen to go straight through the blown-up balloon due to the low amount of material that only scatters a small amount of the IR radiation.

\section{Summary - educational reflections}

In all, we have found IR cameras to be a useful technology in relation to a wide range of thermal phenomena. Given its inherent focus on heat radiation and the temperature of objects towards which it is directed, it turns students' attention to thermal aspects of the surrounding world. In addition, due to the attraction of the technology it encourages students to engage in thermal discovery. We thereby agree with Xie and Hazzard that the technology is particularly well suited to open-ended, inquiry-based approaches to student labs. ${ }^{11}$ However, the technology can also be used in conjunction with more closely guided approaches, such as predictobserve-explain. ${ }^{15}$

Secondary students have been found to use IR cameras as a temperature measurement device. ${ }^{13}$ Our experience with university students, in contrast, is that they rather use IR cameras to investigate thermal properties of different materials as they interact with electromagnetic radiation at different wavelengths.

What limitations are there with IR cameras in physics teaching? First, where handheld IR cameras measure the surface temperature of solids and liquids, traditional thermometers can measure the bulk temperature inside solids, liquids and gases. IR cameras thereby complement thermometers rather than replace them. Second, IR cameras offer a visual approach to macroscopic thermal science, but do not help much in providing microscopic accounts of underlying mechanisms. For instance, students will still have to be taught the role of chemical bonds in phase changes in relation to heat pumps or fire extinguishers, or different atomic 
models for thermal conductivity in order to understand why metals feel cold at room temperature.

Overall, IR cameras advance beyond temperature point-measure technologies such as thermometers and pyrometers. They give students a holistic image that allows them to notice and attend to thermal phenomena, which they most likely would not have seen without them.

\section{References}

1. B. W. Dreyfus, B. D. Geller, D. E. Meltzer, and V. Sawtelle, Resource letter TTSM-1: Teaching thermodynamics and statistical mechanics in introductory physics, chemistry, and biology, Am. J. Phys. 83, 5 (2015).

2. M. E. Loverude, C. H. Kautz, and P. R. L. Heron, Student understanding of the first law of thermodynamics: Relating work to the adiabatic compression of an ideal gas, Am. J. Phys. 70, 137 (2002).

3. M. Prince, M. Vigeant, and K. Nottis, Development of the Heat and Energy Concept Inventory: Preliminary results on the prevalence and persistence of engineering students' misconceptions, J. Eng. Educ. 101, 412 (2012).

4. H. G. Close and R. E. Scherr, Differentiation of energy concepts through speech and gesture in interaction, American Institute of Physics Conference Proceedings 1413, 151 (2012).

5. A. Hofstein and V. N. Lunetta, The role of the laboratory in science teaching: Neglected aspects of research, Rev. Educ. Res. 52, 201 (1982).

6. A. Hofstein and V. N. Lunetta, The laboratory in science education: Foundations for the twenty-first century, Sci. Educ. 88, 28 (2004).

7. D. S. Domin, A review of laboratory instruction styles, J. Chem. Educ. 76, 543 (1999).

8. M. Vollmer and K.-P. Möllmann, Infrared thermal imaging: fundamentals, research and applications (Wiley-VCH, Weinheim, Germany, 2010).

9. M. Vollmer, K.-P. Möllmann, F. Pinno, and D. Karstädt, There is more to see than eyes can detect - Visualization of energy transfer processes and the laws of radiation for physics education, Phys. Teach. 39, 371 (Sept. 2001).

10. The Concord Consortium. Infrared Tube., http://energy.concord.org/ir (Accessed 16 May 2014).

11. C. Xie and E. Hazzard, Infrared imaging for inquiry-based learning, Phys. Teach. 49, 368 (Sept. 2011).

12. J. Haglund, F. Jeppsson, and K. Schönborn, Taking on the heat - a narrative account of how infrared cameras invite instant inquiry, Res. Sci. Educ. (2015).

13. J. Haglund, F. Jeppsson, D. Hedberg, and K. J. Schönborn, Students' framing of laboratory exercises using infrared cameras, Phys. Rev. ST Phys. Educ. Res. 11, 020127 (2015). 
14. K. J. Schönborn, J. Haglund, and C. Xie, Pupils' early explorations of thermoimaging to interpret heat and temperature, J. Balt. Sci. Educ. 13, 118 (2014).

15. R. White and R. Gunstone, Probing understanding (The Falmer Press, London, UK, 1992).

16. W. M. Christensen, D. E. Meltzer, and C. A. Ogilvie, Student ideas regarding entropy and the second law of thermodynamics in an introductory physics course., Am. J. Phys. 77, 907 (2009).

17. J. B. Brown, Thermodynamics of a rubber band, Am. J. Phys. 31, 397 (1963).

18. H. Georgiou, K. Maton, and M. Sharma, Recovering knowledge for science education research: Exploring the "icarus effect" in student work, Canadian Journal of Science, Mathematics and Technology Education 14, 252 (2014).

19. J. Leslie, An experimental inquiry into the nature and propagation of heat (Mawman, London, UK, 1804).

20. R. G. Olson, A note on Leslie's cube in the study of radiant heat, Ann. Sci. 25, 203 (1969).

21. R. A. Bartels, Do darker objects really cool faster?, Am. J. Phys. 58, 244 (1990). 